\title{
La PAC en discussion. Un nouvel outil pour une nouvelle approche
}

Jean-Christophe Kroll

\section{(2) OpenEdition}

1 Journals

\section{Édition électronique}

URL : http://journals.openedition.org/economierurale/2782

DOI : 10.4000/economierurale.2782

ISSN : 2105-2581

\section{Éditeur}

Société Française d'Économie Rurale (SFER)

\section{Édition imprimée}

Date de publication : 1 septembre 2010

Pagination : 123-126

ISSN : 0013-0559

\section{Référence électronique}

Jean-Christophe Kroll, «La PAC en discussion. Un nouvel outil pour une nouvelle approche », Économie rurale [En ligne], 318-319 | juillet-octobre 2010, mis en ligne le 01 octobre 2012, consulté le 30 avril 2019. URL : http://journals.openedition.org/economierurale/2782 ; DOI : 10.4000/

economierurale. 2782 


\title{
- La PAC en discussion Un nouvel outil pour une nouvelle approche
}

\author{
Jean-Christophe KROLL \\ Professeur, Centre d'économie et de sociologie appliquées à l'agriculture et aux espaces ruraux, \\ CESAER, UMR 1041 INRA-AGROSUPDIJON
}

Quelle politique agricole commune après 2013 ? Les contributions au débat sont nombreuses et l'éventail des préconisations large. Jean-Christophe Kroll propose ici un nouvel outil, le paiement contra-cyclique d'agriculture durable. Que représente exactement ce nouvel instrument ? Son utilisation permettrait-elle à la politique agricole d'être plus cohérente face au dogme du découplage et à la dichotomie des deux piliers de la PAC? (NDLR)

U n premier constat empirique et théorique s'impose : le découplage, que l'UE a mis en avant comme le fer de lance de ses réformes successives, est un outil inadapté, coûteux et inefficace.

Initié par les États-Unis en 1996, il a été abandonné dans ce pays dès 2002. Appliqué dans l'Union européenne à partir de 20042005, il a conduit en 2007 à dilapider quelque 7 milliards d'euros sous forme de rente parfaitement injustifiée aux céréaliers européens, alors même que ces derniers profitaient d'une exceptionnelle flambée des prix. Au plan théorique, la littérature fournit de multiples arguments pour démontrer que le cadre mobilisé pour justifier le découplage (existence d'un système complet de marchés concurrentiels parfaitement efficient) repose sur d'hypothèses abusivement simplificatrices et parfaitement irréalistes en agriculture. Dans ce secteur, les marchés des produits et des facteurs sont sujets à de multiples imperfections que de nombreux économistes ont analysées depuis la grande crise des années trente (concurrence imparfaite, dissymétries d'information, incertitude et aversion au risque des agents, etc.), si bien que le libre jeu de marchés instables, loin de conduire à l'optimum, conduit plutôt en agriculture au chaos généralisé, au profit peut-être des spéculateurs, mais à coup sûr au détriment des consommateurs et de l'efficacité des investissements productifs.

L'objet de débat n'est pas d'embrasser toutes les conséquences qu'impliquent ces constats théoriques et empiriques en matière de politique agricole. Cette entreprise fait actuellement l'objet de multiples groupes de travail à l'échelle française et communautaire, dans la perspective d'une nouvelle PAC pour l'après 2013. L'objectif est ici plus restreint, et se focalise sur un point particulier tant théorique que pratique : celui des productions jointes et de la dichotomie entre le premier et le second pilier de la PAC.

\section{Un enjeu spécifique Les productions jointes}

Le dogme du découplage a conduit à isoler les aides directes au revenu, désormais réputées sans effet sur l'orientation des assolements et des manières de produire, dans un premier pilier de la PAC tandis que les aides contractuelles ciblées sur des investisse- 
ments productifs environnementaux ou territoriaux étaient regroupées, à partir de 1999, dans un second pilier, celui du « développement rural ». Il en résulte une conception parfaitement schizophrène de la politique agricole, qui adresse aux producteurs des injonctions totalement contradictoires avec : - un premier pilier de la politique (le FEAGA) ${ }^{1}$ qui, par la dérégulation des marchés et la répartition des aides selon le capital foncier, soutient la concentration des exploitations, la réduction de la maind'œuvre, la spécialisation des systèmes agricoles, la concentration géographique des productions ;

- un second pilier (le FEADER) ${ }^{2}$ qui finance, à l'inverse, de manière volontariste, les efforts de diversification des productions, d'installation et de création d'emplois ainsi que le maintien de l'activité dans les zones difficiles.

Les dangers de cette dichotomie entre le premier pilier et le second se renforcent, au plan théorique, avec la question de la « jointure » entre production de biens marchands et de biens non marchands. La séparation de la rémunération du producteur entre une rémunération par les prix des productions marchandes, et une rémunération par les primes des biens publics produits repose sur une hypothèse lourde : l'indépendance des cycles de production de ces deux types de biens. Or les agronomes savent parfaitement qu'un système de production agricole constitue un tout, au sein duquel les itinéraires techniques s'imbriquent totalement, et qu'il est impossible de séparer les productions marchandes, de celles d'aménités non marchandes au sein des itinéraires de production. Il s'agit à l'évidence de productions intimement jointes. C'est donc à travers la rémunération du produit, que doivent pouvoir se valoriser les aménités liées aux choix des itiné-

1. FEAGA : Fond européen agricole de garantie. 2. FEADER : Fond européen agricole pour le développement rural. raires de production. Comment justifier sinon le maintien d'aides couplées aux vaches allaitantes, comme le concède le règlement communautaire, si ce n'est pas la reconnaissance implicite que la meilleure manière d'entretenir l'espace et de préserver les paysages, c'est d'y faire paître des animaux ?

À partir de ces considérations, apparaît une contradiction radicale entre le projet de développement d'une agriculture durable, et celui d'une agriculture uniquement orientée vers la compétitivité prix, par les signaux du marché. Le développement d'assolements durables suppose par exemple des rotations de cultures longues sur 4, 6 ou 10 ans (complémentarités entre légumineuses et autres cultures, rupture des cycles de développement des parasites), selon un pas de temps parfaitement incompatible avec les exigences d'ajustement à court terme des assolements en fonction du signal des prix. L'investissement dans des itinéraires de production durable à moyen et long terme suppose que cet investissement ne soit pas ruiné par un retournement intempestif des marchés à court terme. Cette assurance de l'investissement dans la durabilité suppose donc une garantie contre les fluctuations à court terme des prix.

\section{Un nouvel outil Le paiement contra-cyclique agriculture durable}

Ces considérations théoriques et pratiques conduisent à proposer un nouvel outil, baptisé provisoirement Payement contra cyclique agriculture durable (PCAD).

Avec le PCAD, la garantie est donnée au producteur, dans un engagement contractuel sur le moyen terme, d'une rémunération minimale de ses produits (prix d'objectif ou target price) au moyen de paiements contra-cycliques. Cette garantie permet à l'investissement, dans des itinéraires de production innovants, de ne pas être ruiné par les retournements 
intempestifs des marchés mais suppose en contrepartie un engagement dans des itinéraires de production réellement durables, qui doit se vérifier à partir d'un certain nombre de critères : apport maximum en moyenne par hectare et par animal de matière azotée en provenance de l'extérieur de l'exploitation ; productivité minimale des consommations intermédiaires (économies d'intrants) mesurée, par exemple, par un ratio minimum de la valeur ajoutée dans le produit brut ; etc. ${ }^{3}$

L'objectif étant de soutenir les économies d'intrants, l'assurance porte bien sur le chiffre d'affaires, et non sur les marges, l'assurance marge restant une incitation au maintien des systèmes coûteux en intrants. Les aides contra-cycliques restent certes couplées, mais ce couplage se justifie théoriquement par la «jointure » et les contraintes environnementales imposées, si bien que le paiement contra-cyclique ne rémunère pas tant la production, que les efforts réalisés pour élaborer cette production de manière durable. D'ailleurs, en cas de défaillance (critères de durabilité non vérifiés), les PCAD se voient réduits en proportion de l'écart à la norme minimale imposée.

La garantie du chiffre d'affaires, par le truchement de prix minimum garantis à la production permet de jouer sur les rapports de prix pour soutenir plus particulièrement les productions utiles à l'environnement et à la durabilité (soja et autres légumineuses par exemple) même si ces productions sont mal valorisées sur le marché. En effet, le critère de compétitivité ne peut plus, dans ce cas, se limiter à une seule compétitivité prix, mais suppose une prise en compte globale multicritères des perfor-

\footnotetext{
3. Les critères ici mentionnés le sont à titre strictement indicatif. L'élaboration du projet supposerait un approfondissement du travail avec les agronomes et les écologues, pour définir des critères qui soient suffisamment pertinents, tout en restant accessibles et mesurables.
}

mances, qui inclut aussi la compétitivité environnementale.

Enfin, la dimension sociale de la durabilité ne peut non plus être ignorée, ce qui suppose un plafonnement des paiements contra-cycliques par exploitation ou, encore mieux, par actif, afin de soutenir en priorité les itinéraires techniques qui valorisent le mieux l'emploi dans la création de valeur ajoutée économique et environnementale.

\section{Une nécessaire articulation aux autres dispositifs}

Ce nouveau concept de paiement contracyclique agriculture durable permet de réconcilier le premier pilier avec le second, en organisant la synergie des financements plutôt que leur gaspillage par double coût (indemniser sur le second pilier les actions entreprises pour corriger les excès soutenus par le premier pilier). Il ne préjuge pas des autres systèmes utiles pour l'ensemble de la production, tels que filets de sécurité, stockage et organisation des marchés. Il peut induire le risque d'un dualisme de l'agriculture, l'agriculture en PCAD évoluant vers des systèmes durables, tandis que l'agriculture hors PCAD renforcerait sa compétitivité économique à partir d'itinéraires techniques rentables à court terme, mais fondés sur une surexploitation et une dégradation accrue des ressources naturelles et de l'emploi. Ce risque peut être contenu par un pilotage judicieux des niveaux de contraintes de base applicables à tous $(\mathrm{BCAE})^{4}$, et des niveaux de contraintes renforcées du PCAD. Bien plus, à l'image de ce qui a pu se faire en Suisse, on peut imaginer un glissement progressif des normes, l'agriculture durable en PCAD inaugurant les itinéraires techniques à généraliser progressivement dans l'ensemble de la production.

4. BCAE : Bonnes conditions agricoles et environnementales. 
On peut aussi imaginer que le PCAD constitue le premier volet d'un « contrat agriculture durable », qui inclurait d'autres exigences spécifiques en matière de biodiversité, de protection d'espaces remarquables etc., faisant l'objet d'un engagement contractuel complémentaire et d'une rémunération spécifique.

Reste qu'au-delà du principe, l'efficacité, la faisabilité et l'acceptabilité du dispositif dépendront largement du réglage des curseurs qui régissent sa mise en œuvre : d'une part les taux de couverture des variations de prix par les PCAD qui, à priori, doivent rester suffisamment élevés pour donner au dispositif une attractivité réelle et, d'autre part, des niveaux de prestations requises et de plafonnement des PCAD notamment, qui doivent être suffisamment contraignants pour rendre le dispositif crédible auprès des contribuables européens. Ce qui laisse une marge de manœuvre importante à la négociation politique.

\section{Un dispositif contractuel volontaire}

Cependant, il importe de souligner que le PCE est un contrat, qui procède d'un choix volontaire, et non d'une obligation réglementaire. Cela signifie que les agriculteurs qui souhaitent rester dans une logique de compétitivité marchande sont libres de leur choix, sous réserve de faire la preuve de leur efficacité économique sans aides publiques spécifiques. Cela signifie que les États membres, qui ne souhaitent pas mobiliser ce nouvel outil, ne sont pas obligés de faire appel à lui. À l'image de ce que permettait le règlement de 1999, la subsidiarité nationale doit pouvoir s'exercer, pour permettre à chaque État membre d'ajuster la répartition des soutiens entre les différentes catégories d'agriculteurs (contractants et non contractants), et entre les différents espaces de production agricole, compte-tenu des problèmes environnementaux et sociaux spécifiques à chaque espace.

\section{Au-delà de l'outil, une exigence de cohérence}

Pour ce dispositif, de multiples mises au point techniques peuvent s'imaginer et l'objectif visé, avec la présentation de ce PCAD purement virtuel, n'est sûrement pas d'introduire une nouvelle querelle byzantine sur les outils, pour occulter à nouveau les enjeux théoriques et politiques de fond. Il s'agit surtout, à partir d'une illustration pratique, de souligner l'urgence de s'affranchir du carcan intellectuel stérilisant que représentent le dogme du découplage et la dichotomie des deux piliers sur lesquels reposent les orientations récentes de la PAC. Il s'agit par là même, de penser un nouveau dispositif dans une vision globale cohérente, afin d'optimiser les dépenses publiques, ce qui est loin d'être le cas, dans la PAC actuelle. 